\title{
A Mini-review of Face Alignment - Classical Method versus Deep Learning Approach
}

\author{
Nabilah Hamzah, Fadhlan Hafizhelmi Kamaru Zaman and \\ Nooritawati Md Tahir
}

\begin{abstract}
Face alignment is one of the vital research areas to be explored specifically face tasks like face recognition, face verification, face reconstruction, and facial expression analysis. Hence, the need for robust face alignment is still in demand. Numerous classic methods have used the 2D image for the detection of facial landmarks but this task is challenging due to several reasons, for instance, large poses, semi-frontal images, and facial expression. Abundant techniques have been established to mitigate all these challenges but there are far from being solved. Hence this mini-review discussed the face alignment methods based on the classic method to the state-of-the-art that includes the 2D-face alignment along with the 3D-face alignment approach. Based on the review done, the 3D model could combat large poses, facial expressions, and semi-frontal images however some of the facial landmarks are not visible and stack together for occluded face images. Hence, this will be the research area to be explored further in ensuring robustness and better accuracy in the face alignment area.
\end{abstract}

Index Terms-Face Alignment, Deep Learning, 2D-face alignment, 3D-face alignment

\section{INTRODUCTION}

$\mathrm{C}$ OMPUTER VISION has become a very popular research area which includes object detection, face recognition, face detection, face alignment, image-to-image translation, text-toimage translation, medical image translation, super-resolution, and many more. Amongst these areas, face alignment is one of the vital areas [1] along with an image-to-image translation, facial expression analysis, and face editing that depends on good face alignment techniques to achieve good performance [2]. Note that face alignment is a process to localize and detect facial landmarks on an image [2].

This manuscript is submitted on $3^{\text {th }}$ June 2021 and accepted on $30^{\text {th }}$ July 2021. The research is funded by the Ministry of Higher Education (MOHE) Malaysia (TRGS) No: 600-IRMI/TRGS 5/3 (001/2019)-1, the Research Management Centre (RMC), Universiti Teknologi MARA (UiTM), College of Engineering UiTM, Selangor, Malaysia, and National Institute of Forensic Medicine, Malaysia for all support given in this study.

Nabilah Hamzah is a post-graduate student from the College of Engineering, Universiti Teknologi MARA (UiTM), Shah Alam, Selangor (email: 2019125215@isiswa.uitm.edu.my ). Fadhlan Hafizhelmi Kamaru Zaman and Nooritawati Md Tahir are lecturers at the College of Engineering, Universiti Teknologi MARA, Selangor. (e-mail: fadhlan@uitm.edu.my / noorita tahir@yahoo.com ).

1985-5389/C 2021 The Authors. Published by UiTM Press. This is an open access article under the CC BY-NC-ND license (http://creativecommons.org /licenses/by-nc-nd/4.0/).
Face alignment is not new in computer vision since face alignment is the first step or a starting point for all face analysis tasks. In addition, face alignment plays an important role in face recognition tasks [3], [4], facial expression recognition [5], and face attributes [6]. In addition, face alignment is also widely used during face recognition analysis to improve and enhance the algorithm against pose variation [7], [8]. Moreover, in facial expression recognition, face alignment is used to extract features of the face based on several facial expressions [9]. Conversely, face attributes tasks such as eyeglasses use specific positions of the face in determining the facial landmarks via the face alignment process [10]. Therefore, studies related to this area are still in demand in ensuring accurate and robust face alignment. Numerous techniques and methods are established to address various problems such as large poses [11], [12], semi-frontal image [13], as well as face expression [14].

Most of the approaches related to face alignment involve poses, semi-frontal image, and facial expression of a $2 \mathrm{D}$-face alignment are still not robust in resolving the challenges encountered, therefore face alignment is evolving from the 2Dface alignment to the 3D-face alignment. Here, the 3D-face alignment focuses on constructing the face to provide a more dense face alignment [13]. With the 3D-face alignment, images with large poses, expressions, and semi-frontal images can be reconstructed [2], [13], [14]. Note that face alignment can be divided into two methods specifically the classic method approaches that include Active Appearance Model (AAM) [15], [16], Active Shape Model (ASM) [17], Constrained Local Model (CLM) [5], [18], [19], cascade regression [20] and the recent technique is the deep learning approach [21], [22], [23]. An example of a classic method is using the cascade classifier, regression technique, and analysis-by-synthesis. As for deep learning (DL), this approach uses the artificial neural network (ANN) deep learning (DL) technique where many models can be fit to solve the face alignment problem.

Hence in this study, a mini-review of the face alignment technique from the classic method followed by the DL approach will be conducted. The evolution of face alignment from the classic method to the most recent technique will be elaborated, reviewed, and discussed accordingly. 


\section{LITERATURE SEARCH OVERVIEW}

In the classical method, the approach elaborates all the techniques used in face alignment excluding the DL technique. As for the DL, it comprised face alignment discussion related to the DL or based on the enhancement of classical method with DL. Article search was done systematically using IEEE Xplore, Google Scholar, and Web of Science (WOS) for over 20 years period of time. The keywords used to search are face alignment model and face alignment deep network. After screening, the total articles obtained are more than 200 articles, and using our protocol of exclusion and inclusion criteria, for the final set of articles, a total of 56 articles are identified. Unlike the recent review from Gogic et al. [24] that only focused on the regression based-model of face alignment, this mini-review focuses on the classical method and DL-based model. Specifically, this mini-review emphasize on the face alignment technique from a 2D to a 3D-based approach. This mini-review discussed various techniques and shows how each of the techniques improves toward years. The scope of this review includes the two most popular methods namely AAM and CLM because these two models are widely used by most researchers to solve the face alignment problem. The literature search began from the initial work done by Cootes et al. [15] using the AAM technique in the year 2001.

For the DL face alignment-based approach, the review comprised of the 2D-face alignment and the 3D-face alignment technique. DL is already known for its ability in solving complex tasks in the computer vision area including face alignment tasks. As mentioned earlier, the 2D-face alignment era is now towards the $3 \mathrm{D}$-face alignment since the $3 \mathrm{D}$-face alignment can solve issues related to large poses and semifrontal image problems. Table 1 shows the two categories related to this mini-review which are classical and DL techniques.

TABLE I

SCOPE OF THE ClASSICAL AND DL TECHNIQUES IN THE MINIREVIEW

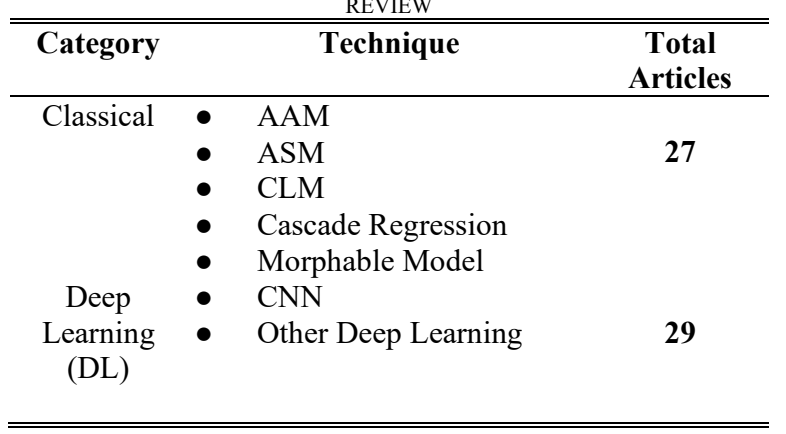

\section{OVERVIEW}

This section will discuss and detail each of the articles under the selection criteria mentioned earlier.

\section{A. Face alignment based on Classical Approaches}

A classical approach is an approach without using artificial intelligence. Even though many classical methods are already being modified and enhanced based on DL or artificial intelligence but for this section, any deep learning approach is excluded while AAM and CLM are discussed as classical methods in this section on how the modeling and fitting are done for each of the methods respectively.

\section{i. Active Appearance Model (AAM)}

The active appearance model or AAM is a linear statistical model proposed by Cootes et al. [25]. This model was widely used in computer vision tasks. In the face alignment field, AAM was utilized for over a decade and the most well-known method was the generative model family [15], [25]-[28].

AAM Modeling. AAM is built by combining the shape model, the appearance model, and the motion model [25], [28]. For the shape model, ground truth labeling is needed to label the landmark points. The landmark points are a $2 \mathrm{D}$ coordinate, where it can be defined as $s_{i}=\left(x_{1}, y_{1}, x_{2}, y_{2}, \ldots \ldots x_{N}, y_{N}\right)$ for each of the training images $\left\{I_{i}\right\}$ and then PCA is applied to obtained the linear shape model. Then the linear shape model can be mathematically derived in Eq (1) [25], [27]:

$$
s=s_{0}+\sum_{i=1}^{M} p_{i} s_{i}
$$

where $s_{0}$ is the mean shape, $p_{i}$ is the shape parameter and $s_{i}$ is the matrix of the landmark's facial point.

The appearance model is obtained by warping each of the training images using the mean shape, $s_{0}$ as the reference frame followed by PCA into the warped appearance [25], [28]. The appearance model is defined in $\mathrm{Eq}(2)$ as:

$$
T(u)=T_{o}(u)+\sum_{i=1}^{I} \lambda_{i} T_{i}(u)
$$

The appearance model is based on the mean shape, $s_{o}$ base mesh where there is a set of pixels $u=(x, y)^{N}$ lies in the base mesh $s_{o}$. Then a set of training images is defined as $T(u)$ over the pixel values $u \in s_{o}$. Since the appearance model is a linear variation, the appearance model, $T(u)$ can be defined as $T_{o}(u)$ plus a linear combination of $l$ appearance images $T_{i}(u)$. The coefficient $\lambda_{i}$ is the appearance parameter.

The motion model is a model to produce a shape-free texture between the images and the ground truth labeling. It is a warp function so that the model points lie on the image points [15].

AAM Fitting. The objective of the AAM fitting is to minimize the sum of the square difference between the appearance model, $T(u)$, and the warp training images that are warp back into the base mesh, $I(N(W(u ; p) ; q))$ [27].

$$
\sum_{u \in T_{o}}\left[T_{o}(u)+\sum_{i=1}^{I} \lambda_{i} T_{i}(u)-I(N(W(u ; p) ; q)\right.
$$

AAM fitting aims to minimize Eq (3). There are two different approaches for AAM fitting. The first approach is to 
learn the relationship between image and parameter using the regression approach. The work from Cootes et al. [25], assumes a linear relationship learned by the linear regression. However, as discussed in [16], a non-linear approach in fitting the model was used and showed improvement in the results. The second approach is to minimize Eq (3) using Lucas-Kanade image alignment [26]. Even though this approach performed faster, but for an unseen image, the algorithm generalized poorly [27].

Advance AAM. AAM has been widely used as a simple framework due to its flexibility [29]. Several types of research that focused to improve AAM performance include work on AAM fitting algorithms that performed well in the constrained dataset [30] but not on the face-in-the-wild dataset. Next, as discussed in [28], using the face-in-the-wild dataset, AAM successfully generalized the unseen images using nonnormalized images pixel as features. Other studies as reported in [28], [31] have improved the model fitting algorithm to determine a more robust AAM.

This section has reviewed the general and the enhancement work on AAM. The classic method is very popular but some enhancements need to be done for improvement in terms of its performance that include AAM efficiency for real-time implementation, lack of robustness under uncontrolled conditions, and less discrimination for segmentation and detection systems [29].

\section{ii. Constrained Local Model (CLM)}

The first work of CLM was proposed by Cristinacce and Cootes where CLM is extended from AAM [19]. CLM is a discriminative approach that utilized a non-rigid registration [18]. CLM used a non-rigid object registration in the training session to independently determine the facial point and optimize the model to determine the best model shape fit during the testing session [18], [32]. CLM can be classified as a discriminative method based on the local detector utilized. In [5] and [33], different types of shape models were used. As elaborated in [5], the CLM used Point Distribution Model (PDM) based shape model. However, the methodology used was similar as in [5] which was based on the combination of the independent local detector with any kind of shape model as CLM.

CLM Modeling. In CLM there are two crucial factors: (i) accurate facial points detection and (ii) a shape model to capture face feature deformations. The detector task is to detect the facial points and provide the probability of the occurrence points at a particular position. The local detector can be further divided into three namely: generative approach, discriminative classifier, and regression-voting approach [34]. A generative approach is an approach that uses image local patches at the annotated facial landmark points. The discriminative classifier approach learns a binary classifier of each facial landmark point with annotated image patches to discriminate whether the target point is aligned or not [5], [35]. On the other hand, the regression-voting approach depends on the vote of the target point from the nearby region followed by computing the probabilities based on the accumulated votes from the different region part [17].
The local detector is typically not very accurate because in training sessions there are many variations and the correct location for facial landmarks will not always be located at the same place. Therefore, the global shape model is required to regularize the detection of the local detector. Conventional CLM used the PDM-based shape model as a global shape model where this model normalized the face shape as multivariate Gaussian followed by approximation using Principal Component Analysis (PCA) [34]. In another work, instead of using the PCA, the ICA-based CLM was used [36].

CLM Fitting. PDM-based CLM is to determine the best shape parameter, $s$ that maximizes the probability of its points corresponding to consistent locations of the facial landmarks. PDM-based CLM can be written as Eq (4) :

$$
\begin{aligned}
& s^{*}=\operatorname{argmax} s\left(s \mid\left\{I_{i}=1\right\}_{i=1}^{N}, I\right) \\
& \quad=\operatorname{argmax} s(s) \prod_{i=1}^{N} s\left(I_{i}=1 \mid x_{i}(s), I\right)
\end{aligned}
$$

where $I$ is the image and $s$ is the optimal shape parameter. $x_{i}(s)$ is the location of the facial landmarks in the $i$ th point generated by the shape model, $I_{i} \in\{1,-1\}$ is the random variable to determine whether the $i$ th point is aligned or not. $s(s)$ is the distribution to be determined by estimating the training data.

CLM fitting PDM-based is an iterative process that involves convolving the local detector with the training image to obtain the response maps and performing the optimization for the global shape model using the response maps.

Advance CLM. Nowadays, the CLM method has been improved based on three aspects: better local detectors, best fitting approach, and using other shape models. Typically, conventional CLM used Support Vector Machine (SVM) [37] and logistic regression [5] to train the local detector. Until recently, other local detectors were proposed, such as Minimum Output Some of Squared Error (MOSSE) [38] and Local Neural Field (LNF) [39] that performed better. These two detectors can extract and detect more complex features using pixel values and produced higher accuracy during detection. For the best fitting approach, Asthana et al. [37] have proposed a novel Discriminative Response Map Fitting (DRMF) where this fitting outperformed the Regularized Landmark MeanShift (RLMS) fitting method [35] in the wild dataset. RLMS fitting method used the Gauss-Newton optimization and DRMF method to estimate the DPM parameter using response maps based texture model [34]. Another improvement was based on using a different type of shape model. The biggest problem with PDM is that the model flexibility is determined using the PCA dimension. Some other researchers improved the CLM using a different type of shape model. For instance, in [36], the ICAbased CLM was used and the model outperformed the PCAbased CLM. Belhumeur et al. proposed combining the output from the local detector with a non-parametric exemplar shape model [40]. Jin and Tan proposed an extended exemplar-based CLM with Hough voting [41]. The main aim is to ensure a stable facial point to be the anchor point to reduce the ambiguity during localizing an unstable facial point. As reported in [34] the eyepoint as the anchor point was proposed since an eye detector is more reliable rather than other facial 
detectors.

This section discussed the general CLM method along with the improvement method using CLM. The disadvantage of the general CLM was the local detector used to detect the facial landmarks was less effective, the fitting models based on the PDM models need to be improved to perform better in the face wild dataset and the shape model that was based on the PCA constrain. Several studies have proposed to overcome the limitations of CLM by focusing on three criteria namely changing the local detector, using a different fitting approach, and using other shape models. It was proven that changing some of these criteria has improved the CLM performance.

\section{B. Face alignment related to Deep Learning Approach}

Deep learning is based on the ANN where the algorithm is inspired by the human brain [42]. The DL is a computational model that consists of many hidden layers to learn the data representation and gather knowledge [43], [44]. Recently, DL has become more popular because there are many open-source platforms such as TensorFlow, PyTorch, Keras, Caffe, and many more that can be utilized to build the deep learning model. Furthermore, DL has a very large community where the codes and libraries can be obtained online such as the Github website. Because of that, DL has become popular in many research areas.

Figure 1(a) shows the ANN for machine learning whilst Figure 1(b) is the architecture for the DL. The architecture difference between machine learning and DL is the machine learning can consist of one or several hidden layers, however, the DL can have more than one hidden layers that also comprised of layers like max-pooling, ReLu, Recurrent, and Fully Connected Layer [42].

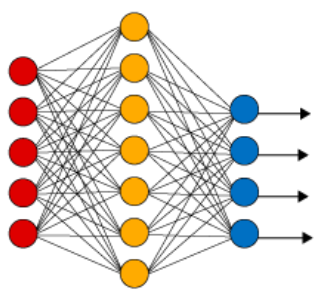

Figure 1(a): Machine Learning Artificial Neural Network[42]

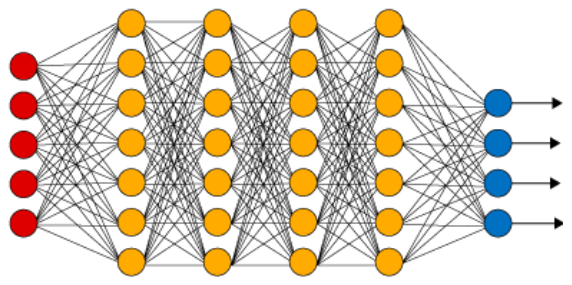

Figure 2(b): Deep Learning Artificial Neural Network [42]

DL especially the Convolutional Neural Network (CNN) is good in extracting image features such as image classification, face recognition, and object detection that has successfully shown good performance based on $\mathrm{CNN}$. CNN can solve a nonlinear relationship between face shape and facial appearance. In the classical approach, most of the techniques used the $2 \mathrm{D}$ face shape for face alignment, however using the DL approach, the $3 \mathrm{D}$-face alignment can be utilized as elaborated below.

2D-Face Alignment. In the face alignment area, numerous studies used the DL to either enhance the classic method with DL or using the DL as a new way for face alignment. However, applying DL directly for face alignment is not significant because the DL like CNN is meant for image classification, and the chance of over-fitting is high. To overcome this, Sun et al. proposed a cascade $\mathrm{CNN}$ where the network is carefully built for the face alignment task [21]. The network consists of a three-layer $\mathrm{CNN}$ and the output of each layer is used for face prediction. The first layer of the network used the face image as input and predict the face shape. Then the other two networks are to define each point of the facial landmarks for better accuracy.

Another work that used a cascade classifier with the DL was Zhang et al. [22] which is the extended work from Sun et al. [21]. In [22], the researcher built a model called the cascade deep model, where this model consists of four layers of a convolutional cascade. Each of the networks was trained to refine the facial landmarks generated by the previous network [22]. Other work on the deep convolutional cascade model was proposed by Dapogny et al. called DeCaFa [23]. DeCaFa model used an end-to-end $\mathrm{CNN}$ with cascade classifier where each of the layers sustained the image spatial resolution throughout the cascade. Between each of the cascade layers, a multiple chained transfer layer with softmax is applied to produce a facial landmarks-wise. Park et al. proposed a combination of heatmap and coordinate regression network with spatial attention [45]. Park et al. implemented combination of different face alignment techniques to boost the model performance namely the accuracy and stability. This method was known as the Attentional Combine Network $(\mathrm{ACN})$ which showed an impressive result in aligning face image with occlusion.

All the above techniques focused on 2D-face alignment but the DL technique is enhanced further for 3D-face alignment. Next, the 3D face alignment technique approach is discussed.

3D-Face Alignment. Most of the techniques focused on the 2D-face alignment that treated the face image as a $2 \mathrm{D}$ object. 2D-face alignment focused on frontal or medium poses. However, if the image in the wild or non-frontal image, facial landmarks detection will be difficult and most of the algorithms only estimated the facial landmarks point [34]. In a 3D-face alignment, a $360^{\circ}$ face orientation can be done while still maintain the correspondence. A 3D-face alignment offers better head pose estimation [46] and facial pose visibility [47]. Hence, the 3D-face alignment can offer a better solution to address the face alignment's large pose problem.

A 3D-face alignment requires a 3D face annotation for facial landmark points. However, most existing face datasets only have the $2 \mathrm{D}$ facial landmarks point annotation without the 3D information. However, a 2D face image can be regarded as a projection of a 3D face on the image plane [48]. From a 2D face shape, a 3D face shape can be constructed using the modelbased structure-from-motion technique [47], [49]. Through this, a 2D face image can be used for 3D-face alignment. Most $3 \mathrm{D}$-face alignment is based on the model regression approach 
for instance cascade regression. In this mini-review, the 3Dface alignment will be divided into two categories; firstly, the $3 \mathrm{D}$-face alignment shape regression and the $3 \mathrm{D}$-face alignment dense model fitting.

\section{i- 3D-Face Alignment Shape Regression}

Captivated by the success of 2D shape regression [50], researchers further explored the face alignment tasks using $3 \mathrm{D}$ shape regression as the 3D-face alignment using either cascade regression technique or CNN that is based on regression approach. The 2D shape regression state-of-the-art shows higher accuracy so the 3D-face alignment inherits the advantage from the 2D shape regression. From a single image, there are three ways to regress it to a 3D face shape. First, by extending the $2 \mathrm{D}$ regression method depth dimension by augmenting the output vector. In [46], the cascade classifier regression approach was used that estimated the facial landmarks in the 3D form and successfully predicted the actual facial landmark's position even though there are occluded due to face rotation. Another work from Valle et al. utilized a coarse-to-fine cascade that assembled the regression tree to a robust face alignment algorithm [51]. Both studies [46], [52] showed that regressing the shape into a $3 \mathrm{D}$ format improved the accuracy and produced a more robust face alignment algorithm for better performance.

Next, is the estimation of the facial landmark points using a 2D face image projection [47], [53]-[55]. Jourabloo and Liu proposed a cascade coupled regressor for estimating both the 2D and 3D facial landmarks automatically [47]. Additionally, the 3D model can be used to estimate the 2D facial landmarks visibilities via surface normal. Conversely, Bittencourt Zavan et al. presented that only the nose region was required as an input to estimate other landmark points and head pose [53]. Based on the nose location, a generic face model is obtained by averaging the training images as either rotated, scaled, or translated into the face image in estimating the head pose. Ruan et al. proposed an end-to-end framework called Self-Aligned Dual face Regression Network (SADRNet) specifically a selfaligned model that generated a 3D output [55]. SADRNet was a combination of two regression modules called pose independent and pose dependent face shape estimation. Addtionally, SADRNet also contain an Attention Network to detect occlusion in the face image and this model was more robust.

Finally, the third approach is based on the 3D-face alignment into two steps: the 2D regression ( $x$ and $y$ ) and the depth regression; $z$ [45], [50], [56], [57]. The first step is to estimate the $x$ and $y$ coordinates for each of the facial landmarks using convolutional heatmap regression. The output from the first step is a $2 \mathrm{D}$ heatmap for each of the facial landmarks. Then, the output of the 2D heatmap and the face image will be the input to the deep network trained via residual learning for regressing the $z$ coordinate.

All third approach in the 3D-face alignment shape regression shows an impressive result in aligning face in 3-dimensional space. Even though some of the models have some deficiencies and disadvantages but mostly achieved a good result in terms of accuracy and stability.

\section{ii- 3D-Face Alignment Dense Model Fitting}

Furthermore, for 3D-face alignment is to consider the 3D surface reconstruction and fitting a dense model to the image. The major problem with the 3D face alignment is the labeling task. As proposed and discussed by [12], the solution for the labeling task is based on the dense 3D face model with cascade $\mathrm{CNN}$ to regress the 3D Morphable Model (3DMM) parameters [58]. The 3DMM is a 3D face textured by transforming the texture and shape of the image into a space vector [59]. The 3DMM using PCA is as described in Eq (5) [12]:

$$
X=\bar{X}+Y_{i d} \alpha_{i d}+Y_{\text {exp }} \alpha_{\text {exp }}
$$

where $X$ is the 3D face and $\bar{X}$ is the mean shape. The $Y_{i d}$ is the identity basis and $Y_{\exp }$ is the expression basis. Next, $\alpha_{i d}$ and $\alpha_{\text {exp }}$ is the shape parameter and expression parameter respectively. Blanz and Vetter are the first to propose the 3DMM algorithm [59] where the classic 3DMM is inefficient and 2D facial landmark point was required before synthesizing [34]. Classic 3DMM is a linear basis model [59], [60], where the linear model can be very limited if there are many types of training data [61]. Due to that problem, many researchers investigated the discriminative fitting approach to improve the classic 3DMM technique using non-linear model fitting [12], [47], [61]. The 3D dense model fitting showed a complete 3D shape face and not only localizing the facial landmark points.

Due to the limitation of the classical 3DMM, Guo et al. proposed a dense regression framework using 3DMM parameters [62]. The enhancement based on the developed regression framework include the stability, speed, and accuracy [12]. The framework outperformed the state-of-the-art framework with 50fps on a single CPU core. Even though this model was a 2D-face alignment approach it has shown better accuracy as compared to the 3D-face alignment approach. Further as reported in [63], Zhu et al. suggested a method beyond 3DMM space specifically the Fine-Grained Reconstruction Network (FGNet) that focused on shape modification by warping the input and output in the UV space.

As compared to the conventional method, the 3D dense model fitting can estimate many more facial landmark points because the number of estimated facial landmarks is dependent on the number produced by the 3DMM parameters. Table 2 detailed the strength and limitation of each technique namely the AAM, CLM and DL.

\section{Discussion}

As stated earlier, two major discussions in this mini-review are the evolvement of the face alignment tasks from the 2Dface alignment to the 3D-face alignment. In addition, the classic method specifically the AAM and CLM methods are discussed and reviewed as well. The drawbacks and limitations of both AAM and CLM are elaborated too.

Conversely, some challenges related to the face alignment process that can be further explored and investigated for better accuracy and performance include (i) dataset acquisition, (ii) multi-task learning, (iii) feature learning, and (iv) 3D-face alignment. 


\section{i. Challenges on data acquisition}

Another challenging part in face alignment is the dataset and annotation of large facial datasets captured in the wild. Nowadays, many standard datasets can be obtained freely online for example Labeled Face in the Wild (LFW), Labeled Face Parts in the Wild (LPFW), UTKFace, YoutubeFace, and many more but, most of the images include occlusions such as facial expression, semi-frontal image, and large poses [34]. Because of these occlusions, it can degrade the face alignment performance. Therefore, many researchers extended the 2Dface alignment to the 3D-face alignment to overcome all the occlusions mentioned above [11], [47], [49], [50], [53]. Even though the $3 \mathrm{D}$-face alignment performed better to overcome the occlusions, some parts of the occlusions cannot be solved. For example, an image with large poses and a semi-frontal image specifically an image that tilts more than $90^{\circ}$ needs to be solved using the 3D-face alignment technique. In addition, several 3D-face alignment models need 3D annotation and not many online datasets can provide this [34]. This is required to train the model using more images as a database and to improve the model performance too.

\section{ii. Multi-task learning}

The multi-task learning goal is to improve the performance of the multiple tasks by learning simultaneously. In face alignment, many factors need to be considered such as large poses, facial expression, and occlusion where all these factors can be a great challenge for multi-tasking. For example, with the face recognition or verification model and face alignment model, this needs to be done separately due to the high computational time required to process both tasks. Some studies utilized the hierarchical method [64]-[66] but mostly omitted the face alignment part. This is because both face alignment and face recognition and verification models required a powerful hardware platform for processing both tasks simultaneously. In other work, instead of increasing the face alignment model performance, the facial landmarks detection technique was improved, and this eventually increased the face alignment performance [22], [67], [68]. These studies showed that the multi-task required to learn the facial landmarks and conducted face alignment too. Results showed that the power of multi-task learning in DL architecture improved the face alignment model performance as compared to a single-task model-based and multi-task learning remains a promising direction for face alignment in future research.

\section{i. $\quad$ Feature Learning}

Feature learning is the most important task in machine learning image analysis. In any face alignment area, feature learning can be treated as shallow feature learning, or using DL has achieved state-of-the-art performance [34]. Feature learning can be utilized to overcome the limitation in face alignment. A classic method relies on a manually labeled image to learn the feature in an image. Conversely, DL approach, the feature learning is automatically done by the DL algorithm [53]. In the DL, the most popular feature learning technique is the CNN [13], [56]. CNN has been widely used and showed good results in extracting crucial features from an image. In addition, for face alignment, $\mathrm{CNN}$ has been used as the feature extractor to extract the facial landmarks for instance the nose, eye, and mouth. $\mathrm{CNN}$ is also used with other techniques to build a good face alignment model. The feature learning technique is important in any face alignment that can handle more occlusion with different scenarios.

\section{ii. 3D-face alignment}

As discussed earlier, most of the classic methods focus on the $2 \mathrm{D}$-face alignment. Note that the $2 \mathrm{D}$-face alignment is mainly built for images with a minimum pose or frontal image [34]. Face orientation varies for each image and images with orientation may lose any of the landmark points. Due to that reason, a 3D-face alignment is proposed as a potential solution. However, there are some limitations and disadvantages related to the 3D-face alignment model. The weakness of the 3D-face alignment is that most of the facial landmarks point was estimated by the developed algorithm [34]. The 3D-face alignment is one of the promising fields in the computer vision task that still deem to be explored for developing a more robust face alignment model.

\section{CONCLUSION}

In conclusion, face alignment models are discussed by highlighting the strength and limitations for each model and based on the mini-review conducted the 3D-face alignment model is the recent approach and performed better as compared to the 2D-face alignment model using either the classical or DL method. In addition, the 3D model can also resolve large poses, facial expression, semi-frontal image occlusion, and robust as well as high precision in aligning the faces under analysis. Even though the $3 \mathrm{D}$-face alignment showed the better result, there is some limitation in this area that include occlusions, for instance, large poses, semi-frontal image, and facial expression since some of the facial landmarks are not visible and stack together. Thus, a 3D annotation is required for initially labeling the facial landmarks. Finally, although face alignment may solve most of the challenges mentioned earlier, face alignment remains an interesting field in computer vision to be explored to produce a much more stable and robust model with better accuracy. 
TABLE 2

SuMmary OF METHOdS USED By RESEARCHERS FOR FACE ALIGNMENT

\begin{tabular}{lcl}
\hline \hline Methods & $\begin{array}{l}\text { Reference } \\
\text { \& Year }\end{array}$ & Methodology Developed \\
\hline $\begin{array}{l}\text { Active } \\
\text { Appearance } \\
\text { Model (AAM) }\end{array}$ & {$[15]-2001$} & $\begin{array}{l}\text { Pioneer works on AAM } \\
\text { using the linear statistical } \\
\text { model approach. }\end{array}$ \\
& {$[26]-2004$} & $\begin{array}{l}\text { An efficient fitting } \\
\text { algorithm based on inverse } \\
\text { compositional. }\end{array}$ \\
& {$[16]-2007$} & $\begin{array}{l}\text { Improvement of the basic } \\
\text { AAM method using a non- } \\
\text { linear discriminative-based } \\
\text { approach. }\end{array}$
\end{tabular}

[28] - 2013 Improvement of AAM using a different fitting model called Active Orientation Model (AOM).

Constrained Local Model (CLM)

Convolutional

Neural

Network

$(\mathrm{CNN})$

$\&$

Other DL
[21] - 2013

[19] - 2006

Development of CLM using different appearance models based on the enhancement of the AAM proposed earlier by [15] to improve the performance in a real-time scenario.

[18]-2008 Optimization of the global detector to improve the CLM efficiency.

[32]-2009 Improve the CLM performance in real-time for non-rigid face alignment.

Utilized a more accurate $\mathrm{CNN}$ for facial landmarks detection.

[69]-2014 The model was developed using the coarse-to-fine auto-encoder network (CFAN) that was cascaded with several stacked autoencoder networks (SAN).

[47] - 2015 Combination of a cascade regression model with DL for detection and aligning the large pose image in the 3D surface.

Strength

Limitation

A flexible model framework comprised of three models specifically the shape, appearance and motion model that was able to enhance the AAM efficiency.

Enhancement of AAM fitting algorithm based on [15].

Enhancement of non-linear discriminative AAM framework as compared to the linear-based approach with an accuracy of $99.7 \%$.

Development of a 2D-face alignment generative model framework based on AAM that was suitable for the pose image with an accuracy of more than $80 \%$.

The global shape model was utilized to regularize the local detector for a more robust face alignment model.

Manage to align all non-rigid objects using the developed optimization algorithm by enforcing convexity at each local patch response surface.

Enhance the non-rigid face alignment with a more simplified optimization algorithm called Exhaustive Local Search (ELS).

Can predict more facial landmarks in semi-frontal or large poses image as compared to the classical method.

Suitable for realization in realtime.

Detection of facial landmarks in large pose images was achieved.
The approximation achieved by the developed algorithm attained an average error of 8.4 as compared to the ground truth.

The mean error attained was $\leq 1.0$ but longer iterations process for computational of the face alignment.

Less effective in analyzing the semi-frontal and large poses images since the training database consists of less than $10^{\circ}$ of rotational images.

Not suitable for large poses and semi-frontal images that further degraded the model performance.

The local detectors used were less effective in detecting facial landmark points.

The linear SVM was used that could not handle the complex problems and the efficiency decreased.

The linear SVM was used that reduced the model performance.

The average error obtained was more than $2 \%$ in detecting 5 points of facial landmarks.

The complexity of the networks contributed to a higher mean average error specifically 5.53 using the HELEN dataset.

The mean average error attained was 4.75 as compared to the ground truth. 
[56]-2016 The CNN was built with heat-map regression based on the fusion of two DL $\mathrm{CNN}$ and residual networks.

[53] - 2016 The nose region was used as the first landmark for detection purposes followed by the head pose estimation.

[54]-2017 Deep Alignment Network (DAN) that consists of multiple stages, where each of the stages has improved the location estimated by the previous stage.

[13]-2018 Reconstruction of the 3D facial structure and dense alignment.

[51] - 2019 The model was developed based on a coarse-to-fine cascade using ensembles of regression trees.
Suitable for detection of facial landmarks in both $2 \mathrm{D}$ and $3 \mathrm{D}$ surfaces using the developed heat-map regression.

Can estimate the head pose with the nose as the main focus.

Detection of facial landmarks in large poses images can be achieved.

Construction of the 3D facial structure and light-weighted network that was independent of any shape model and perform faster.

Suitable for both large face rotation and large pose images.
More complex than other approaches. This method used two deep networks to detect facial landmarks. The average error obtained was $4.6 \%$ as compared to the ground truth point.

Not suitable for semi-frontal and large poses images if the nose is not visible and further decreased the model performance. The average error obtained was more than $10 \%$ as compared to the ground truth point.

The mean error rate attained for estimation of the facial landmarks point was 4.32 using the $300 \mathrm{~W}$ as the dataset.

The mean error rate attained was 3.9625 for facial landmark estimation using the AFLW2000 as the dataset.

The error rate improved for facial landmarks points estimation with an average mean error of 2.26 .

[10] N. Kumar, A. C. Berg, P. N. Belhumeur, and S. K. Nayar, "Attribute and Simile Classifiers for Face Verification," Proc. IEEE Int. Conf. Comput. Vis., no. Iccv, pp. 365-372, 2009.

\section{REFERENCES}

Y. Taigman, M. Yang, M. Ranzato, and L. Wolf, "DeepFace: Closing the gap to human-level performance in face verification," Proc. IEEE Comput. Soc. Conf. Comput. Vis. Pattern Recognit., pp. 1701-1708, 2014.

[2] J. Deng et al., "The Menpo Benchmark for Multi-pose 2D and 3D Facial Landmark Localisation and Tracking," Int. J. Comput. Vis., vol. 127, no. 6-7, pp. 599-624, 2019.

[3] G. J. Edwards, T. E. Cootes, and C. J. Taylor, "Face recognition using active appearance models," Lect. Notes Comput. Sci. (including Subser. Lect. Notes Artif. Intell. Lect. Notes Bioinformatics), vol. 1407, pp. 581-595, 1998.

[4] V. Neagoe and S. M. Ieee, "Face Recognition using PCA versus ICA versus LDA cascaded with the Neural Classifier of Concurrent SelfOrganizing Maps," 2010 8th Int. Conf. Commun., pp. 225-228, 2011.

[5] S. W. Chew, P. Lucey, S. Lucey, J. Saragih, J. F. Cohn, and S. Sridharan, "Person-independent facial expression detection using Constrained Local Models," 2011 IEEE Int. Conf. Autom. Face Gesture Recognit. Work. FG 2011, pp. 915-920, 2011.

[6] Y. Lu, Y. Tai, and C. Tang, "Attribute-Guided Face Generation Using Conditional CycleGAN," ECCV2018, pp. 1-16, 2018.

[7] B. Amos, B. Ludwiczuk, and M. Satyanarayanan, "OpenFace: A General-Purpose Face Recognition Library with Mobile Applications," Tech. Rep. C. C. Sch. Comput. Sci., vol. 16, no. 118, pp. 1-18, 2016.

[8] P. Campadelli, R. Lanzarotti, and C. Savazzi, "A feature-based face recognition system," 12th Int. Conf. Image Anal. Process. 2003.Proceedings., Mantova, Italy, 2003, pp. 68-73, 2003.

[9] I. Cohen, N. Sebe, A. Garg, L. S. Chen, and T. S. Huang, "Facial expression recognition from video sequences: Temporal and static modeling," Comput. Vis. Image Underst., vol. 91, no. 1-2, pp. 160$187,2003$.

[11] X. Yu, J. Huang, S. Zhang, W. Yan, and D. N. Metaxas, "Pose-free facial landmark fitting via optimized part mixtures and cascaded deformable shape model," Proc. IEEE Int. Conf. Comput. Vis., pp. 1944-1951, 2013

[12] X. Zhu, X. Liu, Z. Lei, S. Z. Li, and H. Shi, "Face Alignment Across Large Poses: A 3D Solution," IEEE Conf. Comput. Vis. Pattern Recognit., pp. 146-155, 2016.

[13] Y. Feng, F. Wu, X. Shao, Y. Wang, and X. Zhou, "Joint 3d face reconstruction and dense alignment with position map regression network," Lect. Notes Comput. Sci. (including Subser. Lect. Notes Artif. Intell. Lect. Notes Bioinformatics), vol. 11218 LNCS, pp. 557574, 2018.

[14] F. Liu, D. Zeng, Q. Zhao, and X. Liu, "Joint face alignment and 3D face reconstruction," Lect. Notes Comput. Sci. (including Subser. Lect. Notes Artif. Intell. Lect. Notes Bioinformatics), vol. 9909 LNCS, pp. 545-560, 2016.

[15] T. F. Cootes, G. J. Edwards, and C. J. Taylor, "Active Appearance Models," IEEE Trans. Pattern Anal. Mach. Intell., vol. 23, no. 6, pp. 681-685, 2001.

[16] J. Saragih and R. Goecke, "A nonlinear discriminative approach to AAM fitting," Proc. IEEE Int. Conf. Comput. Vis., 2007.

[17] D. Cristinacce and T. Cootes, "Boosted Regression Active Shape Models," BMVC 2007 - Proc. Br. Mach. Vis. Conf. 2007, 2007.

[18] Y. Wang, S. Lucey, and J. F. Cohn, "Enforcing convexity for improved alignment with constrained local models," 26th IEEE Conf. Comput. Vis. Pattern Recognition, CVPR, 2008.

[19] D. Cristinacce and T. Cootes, "Feature Detection and Tracking with Constrained Local Models," BMVC 2006 - Proc. Br. Mach. Vis. Conf. 2006, pp. 929-938, 2006.

[20] Q. Liu, S. Member, J. Deng, J. Yang, and G. Liu, "Adaptive Cascade Regression Model for Robust Face Alignment," vol. 4, no. 5, 2016.

[21] Y. Sun, X. Wang, and X. Tang, "Deep Convolutional Network Cascade for Facial Point Detection," pp. 0-7, 2013. 
[22] Z. Zhang, P. Luo, C. C. Loy, and X. Tang, "Facial Landmark Detection by Deep Multi-task Learning," Comput. Vis. - ECCV 2014. ECCV 2014. Lect. Notes Comput. Sci., vol. 8694, pp. 94-108, 2014.

[23] A. Dapogny, K. Bailly, and M. Cord, "DeCaFA: Deep convolutional cascade for face alignment in the wild," arXiv, pp. 6893-6901, 2019. I. Gogić, J. Ahlberg, and I. S. Pandžić, "Regression-based methods for face alignment: A survey," Signal Processing, vol. 178, 2021.

[25] T. F. Cootes, G. J. Edwards, and C. J. Taylor, "Active Appearance Models," Lect. Notes Comput. Sci. (including Subser. Lect. Notes Artif. Intell. Lect. Notes Bioinformatics), vol. 1407, pp. 484-498, 1998.

[26] J. Matthews and S. Baker, "Active appearance models revisited," Int. J. Comput. Vis., vol. 60, no. 2, pp. 135-164, 2004.

[27] R. Gross, I. Matthews, and S. Baker, "Generic vs. Person Specific Active Appearance Models," no. 1, pp. 48.1-48.10, 2012.

[28] G. Tzimiropoulos, J. Alabort-I-Medina, S. Zafeiriou, and M. Pantic, "Generic active appearance models revisited," Lect. Notes Comput. Sci. (including Subser. Lect. Notes Artif. Intell. Lect. Notes Bioinformatics), vol. 7726 LNCS, no. PART 3, pp. 650-663, 2013.

[29] X. Gao, Y. Su, X. Li, and D. Tao, "A review of active appearance models," IEEE Trans. Syst. Man Cybern. Part C Appl. Rev., vol. 40 no. 2, pp. 145-158, 2010.

[30] S. Baker, R. Patil, G. Cheung, and I. Matthews, "Lucas-Kanade 20 Years On: An Unifying Framework: Part 5," C. Rep., vol. 56, no. 3, p. 14, 2004.

[31] G. Papandreou and P. Maragos, "Adaptive and constrained algorithms for inverse compositional active appearance model fitting," 26th IEEE Conf. Comput. Vis. Pattern Recognition, CVPR, 2008.

[32] S. Lucey, Y. Wang, M. Cox, S. Sridharan, and J. F. Cohn, "Efficient constrained local model fitting for non-rigid face alignment," Image Vis. Comput., vol. 27, no. 12, pp. 1804-1813, 2009

[33] J. M. Saragih, S. Lucey, and J. F. Cohn, "Face alignment through Subspace Constrained Mean-Shifts," Proc. IEEE Int. Conf. Comput. Vis., no. Clm, pp. 1034-1041, 2009.

[34] X. Jin and X. Tan, "Face Alignment in-the-wild: A Survey," Comput. Vis. Image Underst., vol. 162, pp. 1-22, 2017.

[35] J. M. Saragih, S. Lucey, and J. F. Cohn, "Deformable Model Fitting by Regularized Landmark Mean-Shift," Int. J. Comput. Vis., vol. 91, no. 2, pp. 200-215, 2011.

[36] Q. Zhao et al., "Hierarchical Constrained Local Model Using ICA and Its Application to Down Syndrome Detection," Int. Conf. Med. Image Comput. Comput. Interv., pp. 222-229, 2013.

[37] A. Asthana, S. Zafeiriou, S. Cheng, and M. Pantic, "Robust Discriminative Response Map Fitting with Constrained Local Models," Proc. IEEE Conf. Comput. Vis. pattern recognition., pp. 3444-3451, 2013.

[38] P. Martins, R. Caseiro, and J. Batista, "Non-Parametric Bayesian Constrained Local Models," Proc. IEEE Conf. Comput. Vis. Pattern Recognit., pp. 1797-1804, 2014.

[39] T. Baltrusaitis, P. Robinson, and L.-P. Morency, "Constrained Local Neural Fields for Robust facial landmark detection in the wild," Proc. IEEE Int. Conf. Comput. Vis. Work., pp. 354-361, 2013.

[40] P. N. Belhumeur, D. W. Jacobs, D. J. Kriegman, and N. Kumar, "Localizing Parts of Faces Using a Consensus of Exemplars," IEEE Trans. Pattern Anal. Mach. Intell., vol. 35, no. 12, pp. 2930-2940, 2013.

[41] X. Jin and X. Tan, "Face Alignment by Robust Discriminative Hough Voting," Pattern Recognit., vol. 60, pp. 318-333, 2016.

[42] A. Rosebrock, Deep Learning for Computer Vision with Python, 1st Editio. PYIMAGESEARCH, 2017.

[43] Y. Lecun, Y. Bengio, and G. Hinton, "Deep learning,” Nature, vol. 521, no. 7553, pp. 436-444, 2015.

[44] K. G. Kim, "Deep learning book review," Nature, vol. 29, no. 7553, pp. 1-73, 2019.

[45] H. Park and D. Kim, "ACN: Occlusion-tolerant Face Alignment by Attentional Combination of Heterogeneous Regression Networks," Pattern Recognit., vol. 114, p. 107761, 2021.

[46] S. Tulyakov and N. Sebe, "Regressing a 3D Face Shape from a Single Image," Proc. IEEE Int. Conf. Comput. Vis., pp. 3748-3755, 2015.

[47] A. Jourabloo and X. Liu, "Pose-Invariant 3D Face Alignment," Proc. IEEE Int. Conf. Comput. Vis., vol. 2015 Inter, pp. 3694-3702, 2015.

[48] J. Xiao, S. Baker, I. Matthews, and T. Kanade, "Real-Time Combined 2D+3D Active Appearance Models," Proc. IEEE Comput. Soc. Conf. Comput. Vis. Pattern Recognit., vol. 2, pp. 535-542, 2004.

[49] L. A. Jeni, S. Tulyakov, L. Yin, N. Sebe, and J. F. Cohn, "The First
3D Face Alignment in the Wild (3DFAW) Challenge," Lect. Notes Comput. Sci. (including Subser. Lect. Notes Artif. Intell. Lect. Notes Bioinformatics), vol. 9914 LNCS, pp. 511-520, 2016.

[50] C. Gou, Y. Wu, F. Wang, and Q. Ji, "Shape Augmanted Regression for 3D Face Alignment," Eur. Conf. Comput. Vis., vol. 3, pp. 604$615,2016$.

[51] R. Valle, J. M. Buenaposada, A. Valdés, and L. Baumela, "Face Alignment using a 3D Deeply-Initialized Ensemble of Regression Trees," Comput. Vis. Image Underst., vol. 189, no. October, p. 102846, 2019.

[52] R. Valle, J. M. Buenaposada, A. Valdés, and L. Baumela, "Face Alignment using a 3D Deeply-Initialized Ensemble of Regression Trees," Comput. Vis. Image Underst., vol. 189, no. October, p. 102846, 2019.

[53] F. H. De Bittencourt Zavan, A. C. P. Nascimento, L. P. E. Silva, O. R. P. Bellon, and L. Silva, "3D Face Alignment in the Wild: A Landmark-Free, Nose-Based Approach," Eur. Conf. Comput. Vis. (ECCV 2016), pp. 581-589, 2016.

[54] M. Kowalski, J. Naruniec, and T. Trzcinski, "Deep Alignment Network: A Convolutional Neural Network for Robust Face Alignment," Proc. IEEE Conf. Comput. Vis. Pattern Recognit. Work., pp. 88-97, 2017.

[55] Z. Ruan, C. Zou, L. Wu, G. Wu, and L. Wang, "SADRNet: SelfAligned Dual Face Regression Networks for Robust 3D Dense Face Alignment and Reconstruction," IEEE Trans. Image Process., vol. 30, pp. 5793-5806, 2021

[56] A. Bulat and G. Tzimiropoulos, "Two-Stage Convolutional Part Heatmap Regression for the 1st 3D Face Alignment in the Wild (3DFAW) Challenge," Eur. Conf. Comput. Vis. 2016, pp. 616-624, 2016.

[57] R. Zhao, Y. Wang, C. Fabian Benitez-Quiroz, Y. Liu, and A. M. Martinez, "Fast and Precise Face Alignment and 3D Shape Reconstruction from a Single 2D Image," Eur. Conf. Comput. Vis. (ECCV 2016), pp. 590-603, 2016.

[58] X. Shao et al., "3D Face Shape Regression From 2D Videos with Multi-Reconstruction and Mesh Retrieval," Proc. - 2019 Int. Conf. Comput. Vis. Work. ICCVW 2019, pp. 3090-3095, 2019.

[59] V. Blanz and T. Vetter, "A morphable model for the synthesis of 3D faces," Proc. 26th Annu. Conf. Comput. Graph. Interact. Tech. SIGGRAPH 1999, pp. 187-194, 1999.

[60] S. Romdhani, V. Blanz, and T. Vetter, "Face Identification by Fitting a 3D Morphable Model using Linear Shape and Texture Error Functions," Eur. Conf. Comput. Vis., pp. 3-19, 2002.

[61] L. Tran and X. Liu, "Nonlinear 3D Face Morphable Model," Proc. IEEE Conf. Comput. Vis. pattern recognition., pp. 7346-7355, 2018.

[62] J. Guo, X. Zhu, Y. Yang, F. Yang, Z. Lei, and S. Z. Li, "Towards Fast, Accurate and Stable 3D Dense Face Alignment," Lect. Notes Comput. Sci. (including Subser. Lect. Notes Artif. Intell. Lect. Notes Bioinformatics), vol. 12364 LNCS, pp. 152-168, 2020.

[63] X. Zhu et al., "Beyond 3DMM Space: Towards Fine-Grained 3D Face Reconstruction," Lect. Notes Comput. Sci. (including Subser. Lect. Notes Artif. Intell. Lect. Notes Bioinformatics), vol. 12353 LNCS, pp. 343-358, 2020.

[64] R. Ranjan et al., "A Fast and Accurate System for Face Detection, Identification, and Verification," IEEE Trans. Biometrics, Behav. Identity Sci., vol. 1, no. 2, pp. 82-96, 2019.

[65] H. Li and G. Hua, "Hierarchical-PEP Model for Real-world Face Recognition,” IEEE Access, pp. 4055-4064, 2015.

[66] G. Bai, Y. Zhu, and Z. Ding, "A hierarchical face recognition method based on Local Binary Pattern," Proc. - 1st Int. Congr. Image Signal Process. CISP 2008, vol. 2, pp. 610-614, 2008.

[67] F. J. Chang, A. T. Tran, T. Hassner, I. Masi, R. Nevatia, and G. Medioni, "FacePoseNet: Making a Case for Landmark-Free Face Alignment," Proc. - 2017 IEEE Int. Conf. Comput. Vis. Work. ICCVW 2017, pp. 1599-1608, 2017.

[68] J. Deng, G. Trigeorgis, Y. Zhou, and S. Zafeiriou, "Joint Multi-View Face Alignment in the Wild," IEEE Trans. Image Process. 2019, vol. 28, no. 7, pp. 3636-3648, 2019.

[69] J. Zhang, S. Shan, M. Kan, and X. Chen, "Coarse-to-Fine AutoEncoder Networks (CFAN) for Real-Time Face Alignment," Eur. Conf. Comput. Vis., pp. 1-16, 2014. 


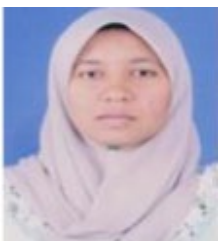

Nabilah Hamzah received B.S degree in Electronic (Computer) Engineering and M.S degree in Science (Electrical Engineering) from Universiti Teknologi Mara (UiTM). From 2014 until now she was a Graduate Research Assistant (GRA) with Universiti Teknologi Mara (UiTM). Her research interest includes in image analysis and Artificial Intelligent (AI).

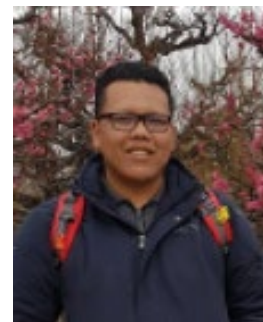

Fadhlan Hafizhelmi Kamaru Zaman received the B.Sc (Hons.) and P.hD. degrees from International Islamic University Malaysia in 2008 and 2015, respectively. $\mathrm{He}$ is currently a Senior Lecturer at Department of Computer Engineering, University of Technology MARA, Malaysia. His research interests are in surveillance system, pattern recognition, signal and image processing, artificial intelligence and computer vision. He is also the Head of Vehicle Intelligence and Telematics Lab. Fadhlan is also a professional Engineer with Board of Engineers Malaysia, and a Chartered Engineer from the Institution of Engineering and Technology, UK

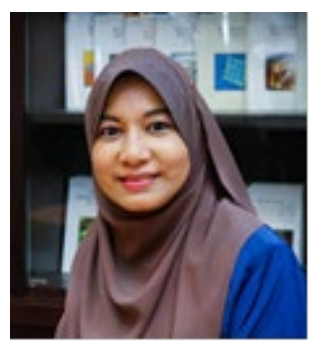

Nooritawati Md Tahir is a lecturer at the College of Engineering, Universiti Teknologi MARA Selangor Malaysia. Her research interest is in the field of Image Processing, Pattern Recognition and Computational Intelligence. She has authored and co-authored more than 250 indexed publications with SCOPUS h-index of 18. In addition, she is a Certified Trainer for Postgraduate Training in Innovation and Entrepreneurship awarded by TCD-UCD Dublin, Ireland, Stanford Faculty Fellow organised by Stanford Technology Ventures Program, Stanford University, US, Chartered Engineer (CEng) from the Institution of Engineering and Technology and Engineering Council UK as well as Senior Member (SMIEEE) of the Institution of Electrical, Electronics Engineer (IEEE). 